\title{
Informe del Cirujano General de 1964 acerca de los daños a la salud atribuibles al consumo de tabaco*
}

A 1 efectuar las valoraciones críticas de los datos e interpretaciones y al formular sus propias conclusiones, el Surgeon General's Advisory Committee on Smoking and Health -los miembros individuales, los subcomités y el Comité como un todo- tomó resoluciones o estableció dictámenes en tres niveles:

I. Dictamen en cuanto a la validez de una publicación o informe. Intervinieron en la elaboración de este dictamen elementos tales como las estimaciones de la competencia y el adiestramiento del investigador, el grado en el que se dejan de lado los prejuicios, el diseño y el alcance de la investigación y la suficiencia tanto de los medios y recursos como de los controles.

II. Dictamen en cuanto a la validez de las interpretaciones de los investigadores con base en sus observaciones y datos, y en lo relativo a la lógica y justificación de sus conclusiones.

III. Dictámenes necesarios para la formulación de conclusiones dentro del Comité.

Las revisiones, análisis y evaluaciones principales de publicaciones e informes sin publicar que contienen datos, interpretaciones y conclusiones de autores las realizaron miembros individuales del Comité y, en algunos casos, consultores. Luego un subcomité revisó y evaluó los planteamientos, a lo que siguió, después de un tiempo adecuado, la consideración crítica por parte del Comité de un informe del subcomité, además de las decisiones relativas a la selección del material que se incluiría en los borradores del informe, junto con los borradores de las conclusiones a las que llegaron los subcomités. Por último, luego de repetidas revisiones críticas de los borradores de los capítulos, el
Comité como un todo formuló y adoptó conclusiones, declarando el dictamen que consideró éste.

No es el fin de esta sección presentar un ensayo sobre la toma de decisiones, ni parece ser necesario describir en detalle los criterios que se utilizaron para establecer juicios científicos en cada uno de los tres niveles mencionados antes. Todos los miembros del Comité se adiestraron en los altos estándares y criterios implícitos en la realización de valoraciones científicas; si algún miembro carecía incluso de una parte pequeña de tal adiestramiento, lo recibía en buena medida de las persistentes discusiones que se originaron en las consultas y en las reuniones de los subcomités y del Comité completo.

\section{Criterios del método epidemiológico}

Sin embargo, es aconsejable explicar de manera breve ciertos criterios que, si bien aplicables a todos los dictámenes implicados en este informe, resultaron en especial importantes en los dictámenes que se basaron en el método epidemiológico. En esta investigación el método epidemiológico se utilizó, de forma extensa, en la valoración de los factores causales en la relación tabaquismo-salud entre seres humanos, sobre quienes no era viable experimentar directamente. La evidencia clínica, patológica y experimental se consideró de modo amplio, y a menudo sirvió para sugerir una hipótesis o confirmar o contradecir otros resultados. Cuando se acoplan con los demás datos, los resultados de los estudios epidemiológicos pueden proporcionar la base sobre la cual es posible establecer dictámenes de causalidad.

Al realizar los estudios mediante el empleo de este método epidemiológico, deben tomarse en cuenta

\footnotetext{
* Nota de los editores: ésta es una traducción del apartado que alude específicamente a los daños a la salud atribuibles al consumo de tabaco; en: U.S. Department of Health, Education, and Welfare. Smoking and Health. Report of the Advisory Committee to Surgeon General of the Public Health Service. Washington, D.C.: U.S. Department of Health Education and Walfare/Public Health Service, 1964; PHS Publication No. 1103.
} 
muchos factores, variables y resultados de investigaciones para determinar, primero, si existe en realidad una asociación entre un atributo o agente y una enfermedad. El dictamen en este punto se basa en medidas indirectas y directas de la asociación que se sugiere. Si se va a demostrar que existe una asociación, surge la pregunta: "¿la asociación tiene un significado causal?"

Los métodos estadísticos no pueden establecer la prueba de una relación causal en una asociación. El significado causal de una asociación es un asunto de juicio que va más allá de cualquier aseveración de probabilidad estadística. Para juzgar o evaluar el significado causal de la asociación entre el atributo o agente y la enfermedad, o efecto sobre la salud, es necesario utilizar varios criterios, aunque ninguno de ellos constituye una base del todo suficiente para el dictamen:

a) La consistencia de la asociación

b) La intensidad de la asociación

c) La especificidad de la asociación

d) La relación temporal de la asociación

e) La coherencia de la asociación

Se recurrió a estos criterios en diversas secciones de este informe. La razón más amplia y clara de su empleo se encuentra, en el capítulo 9, en la sección titulada "Evaluación de la asociación entre el tabaquismo y el cáncer pulmonar".

\section{Causalidad}

Se discutieron con mucha amplitud, en varias reuniones del Comité y de los subcomités, significados y concepciones diversos del término "causa". Las discusiones ocurrieron casi siempre después de que se habían evaluado y estudiado los datos e informes, y en los tiempos en los que se examinaban críticamente las conclusiones y se formulaban las afirmaciones definitivas. Además, emergieron en las mentes de los miembros, de modo constante e inevitable, ideas acerca de la causalidad en el reino de esta indagación, pues les preocupaba el tema que se investigaba -tabaquismo y salud-.

Sin hacer un resumen de los conceptos más importantes de causalidad, que han determinado las actitudes y acciones humanas desde los días incluso anteriores a Aristóteles, pasando por la era continua de observación y experimento, hasta las certidumbres estadísticas de la presente era atómica, es posible establecer brevemente el punto de vista del Comité en lo relativo a la causalidad y al lenguaje utilizado al respecto en este informe:
1. La situación del tabaquismo en relación con la salud de la humanidad incluye un huésped (hombre variable) y un agente complejo (tabaco y sus productos, en particular aquellos que se forman por la combustión al fumar). La prueba de esta investigación se encuentra en el efecto, o no efecto, de los componentes del agente en los tejidos, órganos y diversas cualidades del huésped que podría a) mejorar su bienestar, b) dejarlo proceder en forma normal, o c) afectar su salud de una u otra forma. Para obtener información sobre estos puntos el Comité hizo lo mejor posible, con una amplia ayuda, para examinar todas las fuentes de información disponibles en publicaciones e informes, y mediante consultas con personas bien informadas.

2. Cuando se observó una relación o asociación entre el fumar, u otros usos del tabaco, y alguna condición en el huésped, se evaluó el significado de la asociación.

3. La caracterización de la valoración demandó un término específico, y se consideraron como términos clave "factor", "determinante" y "causa". El Comité acordó que si bien un factor podría constituir una fuente de variación, no todas éstas corresponden a causas. Se acepta que muchas veces es necesaria la coexistencia de varios factores para que ocurra una enfermedad, y que uno de ellos desempeñe tal vez un papel determinante, esto es, sin él los demás factores se vuelven impotentes (como la susceptibilidad genética). Las hormonas en el cáncer de pecho quizá desempeñen uno de estos papeles determinantes. La palabra "causa" es la única en el uso general vinculada con los asuntos que se consideraron en este estudio, y es capaz de transmitir la noción de una relación significativa, eficaz, entre un agente y una alteración o enfermedad asociadas con el huésped.

4. Sin embargo, debe señalarse ahora mismo que ningún miembro del Comité usó la palabra "causa" en un sentido absoluto en el área de este estudio. Si bien varias disciplinas y campos de conocimiento científico estaban representadas entre los miembros, todos compartían una concepción común de la etiología múltiple de los procesos biológicos. Ninguno fue tan ingenuo como para insistir en la monoetiología en los procesos patológicos o en los fenómenos vitales. Todos estaban completamente conscientes de que existe una serie de eventos en las ocurrencias y los desarrollos en estos campos, y que los resulta- 
dos finales constituyen el efecto neto de muchas acciones y reacciones.

5. Admitiendo que se aceptaron estas complejidades, se notará con toda claridad que se afirma la convicción del Comité si se toma en cuenta la decisión que éste tomó respecto del empleo de las palabras "causa", "causa principal", "causa importante" o "asociación causal" en ciertas conclusiones acerca del tabaquismo y la salud.

\section{Capítulo 4}

Esta parte se presenta en dos secciones. La sección A contiene información acerca de los antecedentes, la esencia de los resultados y las conclusiones del Comité sobre salud y tabaco, y una valoración de la naturaleza y magnitud de los riesgos contra la salud. La sección B presenta las conclusiones formales adoptadas por el Comité y los comentarios principales condensados de los resúmenes detallados que aparecen en cada capítulo de la segunda parte del informe. Es posible que el alcance y la profundidad de las indagaciones del Comité sólo se comprenda mediante el estudio del informe completo.

\section{A. Antecedentes y puntos importantes}

En estudios anteriores, el consumo del tabaco, en especial fumar cigarrillos, se ha vinculado causalmente con varias enfermedades: se le ha asociado con el aumento de muertes por tumor maligno de pulmón y otras enfermedades, sobre todo con las relativas a las arterias coronarias, la bronquitis crónica y el enfisema. Estos resultados, que se han divulgado con amplitud y que en la década pasada fueron causa de una gran preocupación pública, han sido aceptados en gran número de países por las instituciones de salud, las asociaciones médicas y las organizaciones de salud de ayuda voluntaria.

El riesgo potencial es grande debido a que estas enfermedades son las principales causas de muerte e incapacidad. En 1962, más de 500000 personas en los Estados Unidos de América (EUA) murieron por enfermedades cardiacas arterioescleróticas (principalmente la enfermedad de las arterias coronarias), 41000 , por tumor maligno de pulmón, y 15 000, por bronquitis y enfisema.

La cantidad de muertes causadas por algunas enfermedades importantes, que se dicen relacionadas con el consumo del tabaco, se muestran en el cuadro I, el cual presenta un aspecto de la magnitud del riesgo potencial; se analizará después el grado de asociación con el uso del tabaco.
Otra causa de preocupación es que las muertes producto de algunas de estas enfermedades se han incrementado con gran rapidez en las últimas décadas.

Las defunciones por tumor maligno de pulmón, inferiores a 3000 en 1930, aumentaron hasta 18000 en 1950. En el corto periodo a partir de 1955, las muertes por esta enfermedad ascendieron desde menos de 27000 hasta 41000 en 1962, un aumento extraordinario que no se había registrado para el tumor maligno en cualquier otro lugar del cuerpo. Si bien, parte de la tendencia creciente del tumor maligno de pulmón se atribuye a mejoras en el diagnóstico y a cambios en la composición por edad y el tamaño de la población, la evidencia deja muy poca duda de que ha ocurrido un verdadero incremento en este padecimiento.

Las muertes debidas a enfermedades del corazón por arterioesclerosis, dolencias coronarias y daños degenerativos aumentaron de 273000 en 1940 a 396000 en 1950, y a 578000 en 1962.

Las muertes registradas por causa de bronquitis crónica y enfisema se incrementaron de 2300 en 1945 a 15000 en 1962.

Los patrones cambiantes y el grado en el consumo del tabaco constituyen un aspecto pertinente del problema tabaquismo-salud.

Cerca de 70 millones de personas en los EUA consumen tabaco en forma regular. Dicho consumo en ese país ha crecido de manera marcada desde el inicio del siglo XX, cuando el consumo per capita era de menos de 50 cigarros al año. A partir de 1910, año en el cual el

\section{Cuadro I \\ Muertes POR CLASE DE ENFERMEDAD. Estados Unidos de AmÉRICA, 1962}

Causa de muerte*

Total Hombres Mujeres

Enfermedadad cardiovascular ateroescle-

rótica degenerativa incluida la enfermedad

coronaria $(420,422) \quad 577918 \quad 348604 \quad 229314$

Enfermedad cardiaca hipertensiva (440-3) $\quad 62176 \quad 26654 \quad 35622$

\begin{tabular}{lllr}
\hline Tumor maligno de pulmón (162-3) & 41376 & 35312 & 6064
\end{tabular}

\begin{tabular}{llll}
\hline Cirrosis del hígado (581) & 21824 & 14329 & 7495
\end{tabular}

\begin{tabular}{llll}
\hline Bronquitis y enfisema $(502,527.1)$ & 15104 & 12937 & 2167
\end{tabular}

\begin{tabular}{lllll}
\hline Ulceras estomacal y duodenal (540-1) & 12228 & 8836 & 3392
\end{tabular}

\begin{tabular}{llll}
\hline Tumor maligno de la vejiga (181) & 8081 & 5575 & 2500
\end{tabular}

Tumor maligno de la cavidad oral (140-8) $\quad 6481 \quad 4920 \quad 1561$

Tumor maligno del esófago (150) $\quad 5088 \quad 3973 \quad 1115$

\begin{tabular}{lllll}
\hline Tumor maligno de la laringe (161) & 2417 & 2172 & 245
\end{tabular}

\begin{tabular}{lllll}
\hline Todas las causas anteriores & 752603 & 403312 & 280 & 381 \\
\hline
\end{tabular}

\begin{tabular}{lllll}
\hline Las demás causas & 1004027 & 531477 & 472550
\end{tabular}

\begin{tabular}{lllll}
\hline Todas las causas & 1756720 & 994739 & 761831
\end{tabular}

* N úmeros de la clasificación estadística internacional entre paréntesis 
consumo por persona (mayores de 15 años) correspondía a 138, éste ascendió hasta 1365 en 1930, 1828 en 1940, 3322 en 1950, y a un máximo de 3986 en 1961. El censo de población de 1955 en los EUA indicó que de la población mayor de 18 años, $68 \%$ de los hombres y $32.4 \%$ de las mujeres fumaban cigarrillos de manera regular.

En contraste con este claro incremento en el consumo de cigarrillos, disminuyó en ese periodo el uso per capita de tabaco en otras formas. El consumo de puros per capita declinó de 117 en 1920 a 55 en 1962. El consumo de tabaco en pipa, que alcanzó un máximo de $21 / 2$ libras por persona en 1910, disminuyó hasta un poco más de media libra por persona en 1962. El uso de tabaco mascado ha descendido de cerca de cuatro libras por persona en 1900 a media libra en 1962.

Los antecedentes para el estudio del Comité han incluido gran cantidad de información general y de resultados de investigaciones previas, que asociaban el aumento en el consumo de cigarrillos con el número creciente de muertes en varios tipos de enfermedades mayores. En este escenario fue donde el Comité inició su trabajo de valoración de la naturaleza y la magnitud de los riesgos para la salud, atribuibles al tabaquismo.

\section{Tipos de evidencia}

Para dictaminar si el fumar y otros usos del tabaco resultan dañinos para la salud, o si se relacionan con enfermedades específicas, el Comité evaluó tres tipos principales de evidencias científicas:

1. Experimentos con animales. En numerosos estudios, los animales se han expuesto al humo y a los alquitranes del tabaco, así como a los diversos compuestos químicos que estos contienen. Siete de ellos (compuestos aromáticos policíclicos) se han señalado como generadores de tumor maligno (cancerígenos). Otras sustancias en el tabaco y el humo, si bien no cancerígenos por sí solos, estimulan la producción de esta enfermedad o reducen el umbral correspondiente a un cancerígeno conocido. Varios gases tóxicos o irritantes, contenidos en el humo del tabaco, producen experimentalmente los tipos de daño no canceroso que se observan en tejidos y en células de fumadores empedernidos, lo cual incluye la supresión de la actividad ciliar que normalmente limpia la tráquea y los bronquios, el daño en los alvéolos pulmonares, y el deterioro en las glándulas mucosas y en las células de copa que producen moco.

2. Estudios clínicos o de autopsia. Las observaciones de miles de pacientes, y de estudios de autopsias de fumadores y no fumadores, muestran que en los primeros ocurren con mayor frecuencia y severidad muchos tipos de daños en las funciones corporales y en los órganos, células y tejidos. Tres tipos de cambios celulares -pérdida de células ciliadas, engrosamiento (más de dos capas de células basales), y la presencia de células atípicas- son mucho más comunes en la capa de revestimiento (epitelio) de la tráquea y los bronquios de fumadores de cigarro que en la de los no fumadores. Algunas de las lesiones avanzadas que se observan en los bronquios de fumadores de cigarrillos son con toda probabilidad premalignas. Los cambios celulares que se descubren por lo común en las autopsias de pacientes con bronquitis crónica, se presentan con mayor frecuencia en los bronquios de los fumadores que en los de no fumadores. Los cambios patológicos en los alvéolos y otros tejidos funcionales del pulmón (parénquima) tienen una asociación bastante cercana con la historia pasada del consumo de cigarros.

3. Estudios de población. Otro tipo de evidencia, en cuanto a la asociación entre tabaquismo y enfermedad, proviene de estudios epidemiológicos.

En estudios retrospectivos, las historias de tabaquismo de personas con una enfermedad específica (por ejemplo, tumor maligno de pulmón) se comparan con las de grupos de control adecuados, sin la enfermedad. Unicamente para el tumor maligno de pulmón, se han efectuado 29 de estos estudios retrospectivos en los últimos años. A pesar de muchas variaciones en el diseño y en el método, al menos uno (relacionado con mujeres) demostró que se encuentran proporcionalmente más fumadoras de cigarro entre las pacientes con tumor maligno de pulmón que en las poblaciones de control sin dicho padecimiento.

Estudios retrospectivos extensos de la frecuencia de síntomas y signos específicos -tos crónica, producción de esputo, insuficiencia respiratoria, dolencias en el pecho y disminución de la función pulmonarmuestran de modo consistente que éstos ocurren con mayor frecuencia en fumadores de cigarro que en no fumadores. Algunos de estos signos y síntomas son las expresiones clínicas de la bronquitis crónica, y otros se asocian más con el enfisema; en general, aumentan con la magnitud del tabaquismo y disminuyen luego de dejar de fumar.

Otro tipo de evidencia epidemiológica en la relación entre tabaquismo y mortalidad surge de siete estudios prospectivos, que se llevaron a cabo a partir de 1951 y en los cuales un gran número de hombres fueron cuestionados acerca de sus hábitos de fumar o no 
fumar. Se obtuvieron los certificados de defunción de aquellos que fallecieron luego de incorporarse en los estudios, lo que permitió calcular las tasas de mortalidad total y de mortalidad de fumadores por causa de diversa índole, así como de no fumadores. Los estudios prospectivos añaden, por ello, varias dimensiones importantes a la información acerca del problema tabaquismo-salud; sus datos posibilitan comparaciones directas de las tasas de mortalidad de fumadores y no fumadores en ambos casos, respecto de causas de muerte generales e individuales, e indican la intensidad de la asociación entre tabaquismo y enfermedades específicas.

Estas tres líneas de evidencia se evaluaron y luego se consideraron en conjunto para extraer conclusiones. El Comité estaba consciente de que no era suficiente el mero establecimiento de una asociación estadística entre el consumo del tabaco y un padecimiento. La pregunta crucial la constituye la importancia causal del uso del tabaco en relación con la enfermedad. En cuanto a tales dictámenes, resultan esenciales las tres líneas de evidencia, como se analiza con mayor detalle en los párrafos anteriores y al inicio del informe.

La evidencia experimental, clínica y patológica, así como los datos de los estudios de población, se subrayan en el apartado de comentarios y conclusiones, que refiere a su vez al lector a los lugares específicos de la parte II del informe, donde se presenta la evidencia pormenorizada.

En los párrafos que siguen, el Comité ha optado por resumir los resultados de los siete estudios de población prospectivos que, como se indicó antes, constituyen sólo un tipo de evidencia, ilustran la naturaleza y la magnitud potencial del problema tabaquismosalud, y sacan a relucir varios factores implicados.

\section{Evidencia de los resultados combinados de estudios prospectivos}

El Comité examinó por separado los siete estudios prospectivos junto con sus resultados combinados. Se asignó un peso considerable a la consistencia de los resultados entre los diversos estudios. Sin embargo, para simplificar la presentación, sólo se destacan aquí los resultados combinados.

De los 1123000 hombres que intervinieron en los siete estudios prospectivos, mismos que proporcionaron historias útiles de hábitos de consumo de cigarro (y otras características, como la edad), 37391 murieron durante los meses o años subsecuentes a los estudios. En la actualidad no se dispone de ningún análisis de datos de estudios prospectivos correspondientes a mujeres.
Para posibilitar la comparación rápida de la mortalidad entre fumadores y no fumadores, se usan de manera amplia dos conceptos en los estudios -el exceso de muertes de fumadores en comparación con las de no fumadores, y la tasa de mortalidad. Después de ajustes de las diferencias en edad y el número de fumadores y no fumadores de cigarrillo, se obtiene un número esperado de muertes de fumadores, con base en las muertes entre no fumadores. El exceso de muertes corresponde entonces al número de muertes reales (observadas) de fumadores que supera al número esperado. La tasa de mortalidad, para la cual se describe el método de cálculo en el capítulo 8, mide las tasas de muerte relativa de fumadores y no fumadores. Si son iguales las tasas de muerte con la edad ajustada, la tasa de mortalidad será igual a 1.0; si las tasas de muerte de fumadores duplican a la de no fumadores, la tasa de mortalidad corresponderá a 2.0. (Expresado como un porcentaje, este ejemplo equivaldría a un aumento de $100 \%$.)

El cuadro II presenta los datos acumulados y combinados respecto de 14 clases de enfermedades para las cuales la tasa de mortalidad de fumadores de cigarro, respecto a la de no fumadores, fue de $1.5 \mathrm{o}$ mayor.

La tasa de mortalidad para las mujeres fumadoras de cigarro, en comparación con la de las no fumadoras, para todas las causas de muerte consideradas en conjunto, equivale a 1.68 , lo que representa una tasa

\section{Cuadro II* \\ MUERTES ESPERADAS Y OBSERVADAS DE FUMADORES SÓLO DE CIGARRILLOS Y TASAS DE MORTALIDAD EN SIETE ESTUDIOS PROSPECTIVOS}

Causa de muerte implícita Muertes Muertes Tasa de esperadas observadas mortalidad

\begin{tabular}{lrrr} 
Tumor maligno de pulmón (162-3) & 170.3 & 1833 & 10.8 \\
\hline Bronquitis y enfisema (502,521.1) & 89.5 & 546 & 6.1 \\
\hline Tumor maligno de la laringe (161) & 14.0 & 75 & 5.4 \\
\hline Tumor maligno oral (140-8) & 37.0 & 152 & 4.1 \\
\hline Tumor maligno del esófago (150) & 33.7 & 113 & 8.4 \\
\hline Ulceras estomacal y duodenal (540, 541) & 105.1 & 294 & 2.8 \\
\hline O tras enfermedades circulatorias (451-68) & 254.0 & 649 & 2.6 \\
\hline Cirrosis del hígado (581) & 169.2 & 379 & 2.2 \\
\hline Tumor maligno de la vejiga (181) & 111.6 & 216 & 1.9 \\
\hline Enfermedad de las arterias coronarias (420) & 6430.7 & 11 177 & 1.7 \\
\hline O tras enfermedades del corazón (421-2.430-4) & 526.0 & 868 & 1.7 \\
\hline Enfermedad cardiaca hipertensiva (440-3) & 400.2 & 631 & 1.5 \\
\hline Arterioesclerosis general (450) & 210.7 & 310 & 1.5 \\
\hline Tumor maligno de riñón (130) & 79.0 & 120 & 1.5 \\
\hline Todas las causas & 15 653.9 & 23223 & 1.68 \\
* Condensado del cuadro 26, capítulo 8, Mortalidad & & \\
₹ N úmeros de la clasificación estadística internacional entre paréntesis \\
§ Incluye todas las otras causas de muerte, así como las listadas antes
\end{tabular}

$\S$ Incluye todas las otras causas de muerte, así como las listadas antes 
de muerte total casi $70 \%$ superior que la de no fumadoras. (Esta tasa incluye tasas de muerte correspondientes a enfermedades que no se listan en el cuadro, así como las 14 clases indicadas.)

En los resultados combinados de los siete estudios, la tasa de mortalidad de fumadores de cigarro respecto a la de no fumadores resultó en particular alta para varias enfermedades: tumor maligno de pulmón (10.8), bronquitis y enfisema (6.1), tumor maligno de la laringe (5.4), tumor maligno de la cavidad oral (4.1), tumor maligno del esófago (3.4), úlcera péptica (2.8) y el grupo de otras enfermedades circulatorias (2.6). En el caso de la enfermedad de las arterias coronarias, la tasa de mortalidad correspondió a 1.7. (cuadro II)

Expresada en forma de porcentaje, esta cifra es equivalente a la afirmación de que en la enfermedad de las arterias coronarias, la principal causa de muerte en los EUA, la tasa de muerte es $70 \%$ superior para los fumadores de cigarro. En cuanto a la bronquitis crónica y el enfisema, que se encuentran entre las principales causas de incapacidad seria, la tasa de muerte para fumadores de cigarro es $500 \%$ más alta que la de no fumadores. En el caso del cáncer de pulmón, la parte del cuerpo donde se presenta con mayor frecuencia el tumor maligno en el hombre, la tasa de muerte es casi $1000 \%$ superior.

\section{Otros resultados de los estudios prospectivos}

En general, cuanto mayor es el número de cigarros fumados diariamente, tanto más alta resulta la tasa de muerte. De acuerdo con los siete estudios prospectivos, en el caso de los hombres que fuman menos de 10 cigarros diarios, la tasa de muerte por todas las causas es casi $40 \%$ más alta que la de los no fumadores. Para aquellos que fuman de 10 a 19 cigarros al día, la tasa es $70 \%$ superior, y para los que consumen de 20 a 39 cigarros, $90 \%$ más alta; la tasa resulta $120 \%$ mayor en el caso de los que superan los 40 cigarros diarios.

Los fumadores de cigarro que habían dejado de fumar antes de participar en los siete estudios presentan una tasa de muerte casi $40 \%$ por arriba de la de los no fumadores, en contraste con la tasa $50 \%$ superior de los que siguen fumando. Los hombres que empiezan a fumar antes de los 20 años de edad tienen una tasa de muerte sustancialmente superior que la de los que lo hacen después de los 25 años. En comparación con los no fumadores, el riesgo de mortalidad de los fumadores de cigarro, después de los ajustes por la diferencia en edad, aumenta con la duración del tabaquismo (número de años), y resulta superior en los que interrumpen el hábito después de los 55 años al de aquellos que lo hacen a una edad menor.

En los dos estudios que registraron el grado de inhalación, la tasa de mortalidad para un nivel determinado de tabaquismo fue mayor para los inhaladores que para los no inhaladores.

La proporción de las tasas de muerte entre fumadores y no fumadores es más alta en las edades más tempranas (40-50) representadas en estos estudios, y declina con el incremento de la edad del fumador.

También se investigaron en los siete estudios las relaciones posibles de las tasas de muerte y otras formas de uso del tabaco. Las tasas de muerte para hombres que fuman menos de cinco cigarros diarios es casi la misma que las correspondientes a no fumadores. En el caso de hombres que fuman más de cinco cigarros al día, las tasas son un poco superiores. Hay ciertos indicios de que estas tasas de muerte más altas ocurren sobre todo en hombres que han fumado más de 30 años y que inhalan el humo hasta cierto grado. Las tasas de muerte para los fumadores de pipa son apenas, y no siempre, superiores a las de no fumadores, incluso en el caso de hombres que fuman 10 o más pipas llenas al día y de aquellos que han fumado pipa durante más de 30 años.

\section{Número excesivo de muertes}

Varios de los informes publicados con anterioridad acerca de los estudios prospectivos, incluyen una tabla que muestra la distribución de los números del exceso de muertes de fumadores de cigarro entre las principales causas de fallecimiento. El riesgo debe medirse no únicamente por la tasa de mortalidad en fumadores y no fumadores, sino también por la importancia de una enfermedad particular como causa de muerte.

En los siete estudios, la enfermedad de las arterias coronarias es el principal contribuyente al exceso de muertes de fumadores y no fumadores de cigarro, con el tumor maligno de pulmón casi siempre en segundo lugar. En la totalidad de los siete estudios combinados, la enfermedad de las arterias coronarias (con tasa de mortalidad de 1.7) corresponde a $45 \%$ de las muertes excesivas entre fumadores de cigarro, en tanto que el tumor maligno de pulmón (con una tasa de 10.8) equivale a $16 \%$.

Algunas otras enfermedades que contribuyen a las tasas de muerte más altas para fumadores y no fumadores de cigarro son las del corazón y las de los vasos sanguíneos -aparte de la enfermedad de las arterias coronarias-, $14 \%$; tumor maligno en otras partes 
del cuerpo -además del pulmón-, $8 \%$; y la bronquitis crónica y el enfisema, $5 \%$.

Puesto que estas enfermedades como grupo son responsables de más de $85 \%$ de la tasa de muertes superior entre fumadores de cigarro, resultan de particular interés para las autoridades de salud pública y la profesión médica.

\section{A sociaciones y causalidad}

El conjunto de información de los estudios prospectivo y retrospectivo de fumadores y no fumadores establece con claridad una asociación entre el tabaquismo y las tasas de muerte sustancialmente más elevadas. Las tasas de mortalidad en el cuadro II proporcionan un índice aproximado del grado de intensidad relativa de esta asociación, para todas las causas de muerte y para 14 clases de enfermedades.

En esta investigación, el método epidemiológico se empleó extensamente en la valoración de los factores causales en la relación del tabaquismo y la salud entre seres humanos, sobre quienes no era viable efectuar experimentación directa. La evidencia clínica, patológica y experimental se consideró por completo y sirvió a menudo para sugerir una hipótesis o para confirmar o contradecir otros resultados. Cuando se vinculan con los demás datos, los resultados de los estudios epidemiológicos pueden proporcionar la base sobre la cual es posible formular dictámenes de causalidad.

Se acepta que no es probable que exista una relación de causa-efecto simple en un producto complejo, como fumar tabaco, y una enfermedad específica en el variable organismo humano. Se reconoce también que muchas veces se requiere la coexistencia de muchos factores para la ocurrencia de una enfermedad, y que uno de ellos desempeñe quizá un papel determinante; es decir, sin él, los demás factores (tales como la susceptibilidad genética) rara vez conducen a la aparición de la enfermedad.

\section{Los efectos del tabaquismo: principales resultados}

El tabaquismo se asocia con un aumento de $70 \%$ en las tasas de muerte por edad específica en hombres. No es posible estimar con exactitud el número total del exceso de muertes relacionadas causalmente con el tabaquismo en la población estadounidense. En vista de la evidencia continua y creciente de muchas fuentes, el dictamen del Comité es que el tabaquismo contribuye de modo sustancial a la mortalidad por ciertas enfermedades específicas y a la tasa de fallecimientos total.

Tumor maligno de pulmón

El tabaquismo se relaciona causalmente con el tumor maligno de pulmón en hombres; la magnitud del efecto del tabaquismo supera por mucho todos los demás factores. Los datos relativos a mujeres, aunque menos extensos, apuntan hacia la misma dirección.

El riesgo de adquirir tumor maligno de pulmón aumenta con la duración del tabaquismo y con el número de cigarros fumados al día, y disminuye al dejar de fumar. En comparación con los no fumadores, los hombres fumadores de cigarro promedio tienen aproximadamente de 9 a 10 veces más riesgo de desarrollar tumor maligno de pulmón, y los fumadores empedernidos, al menos 20 veces más.

El riesgo de desarrollar tumor maligno del pulmón en el caso del grupo combinado de fumadores de pipa, fumadores de cigarro y fumadores de pipa y cigarro es mayor que el de los no fumadores, pero mucho menor que el de los que sólo fuman cigarro.

El tabaquismo es mucho más importante que las exposiciones ocupacionales en las causas de tumor maligno de pulmón en la población en general.

\section{Bronquitis crónica y enfisema}

El tabaquismo es la más importante de las causas de bronquitis crónica en los EUA, e incrementa el riesgo de muerte por bronquitis crónica y enfisema. Existe una relación entre el tabaquismo y el enfisema, pero no se ha establecido que la relación sea causal. Los estudios demuestran que las defunciones por causa de esta última enfermedad resultan muy poco frecuentes en el caso de los no fumadores.

Para el grueso de la población de los EUA, la importancia relativa del tabaquismo como una causa de enfermedad broncopulmonar crónica es mucho mayor que la contaminación atmosférica o las exposiciones ocupacionales.

\section{Enfermedades cardiovasculares}

Se ha establecido que los hombres fumadores de cigarro tienen una tasa de muerte superior por la enfermedad de las arterias coronarias que los no fumadores. Si bien no se ha demostrado el papel causal del tabaquismo en las muertes por el padecimiento anterior, el Comité considera más prudente suponer, desde la perspectiva de la salud pública, que la asociación establecida tiene 
un significado causal, en vez de suspender el dictamen hasta que no quede ninguna incertidumbre.

A pesar de que no se ha establecido la relación causal, la mortalidad más alta de fumadores de cigarro se asocia con muchas otras enfermedades cardiovasculares, que incluyen enfermedades circulatorias diversas, otras enfermedades del corazón, la enfermedad cardiaca hipertensiva y la arterioesclerosis general.

\section{Otras partes del cuerpo donde se presenta cáncer}

Fumar pipa parece relacionarse de manera causal con el tumor maligno de labio; el tabaquismo es un factor importante en la causalidad del tumor maligno de laringe; la evidencia apoya la creencia de que existe una asociación entre el consumo de tabaco y el tumor maligno de esófago, así como entre el tabaquismo y el tumor maligno de la vejiga urinaria en hombres, aunque los datos no resultan suficientes para afirmar que estas relaciones son causales; los datos acerca de la asociación entre fumar y el tumor maligno de estómago son contradictorios e incompletos.

\section{El hábito del tabaco y la nicotina}

El hábito del uso del tabaco se relaciona fundamentalmente con impulsos psicológicos y sociales, reforzados y perpetuados por los efectos farmacológicos de la nicotina.

La estimulación social parece desempeñar un papel central en las primeras experiencias de los jóvenes con el tabaquismo. Ninguna evidencia científica apoya la hipótesis popular de que el tabaquismo entre los adolescentes es una expresión de rebelión contra la autoridad. El estrés individual parece vincularse más con las fluctuaciones en la cantidad que se fuma que con la frecuencia con la que se hace. La evidencia aplastante indica que el tabaquismo -su inicio, hábito e interrupción ocasional- se determina en gran medida por aspectos psicológicos y sociales.

La nicotina se transforma rápidamente en el cuerpo en sustancias relativamente inactivas con baja toxicidad. La toxicidad crónica de pequeñas dosis de nicotina es baja en animales experimentales. Estos dos hechos, cuando se consideran en conjunto con las bajas tasas de mortalidad de los fumadores de pipa y cigarro, indica que es muy baja la toxicidad crónica de la nicotina en cantidades absorbidas al fumar y mediante otros usos del tabaco, y que probablemente no representa un riesgo importante para la salud.

Los efectos benéficos significativos del tabaquismo ocurren fundamentalmente en el área de la salud mental, y el hábito se origina en una búsqueda de satisfacción. Puesto que no es claro ningún medio para medir el grado de estos beneficios, el Comité no encontró bases para un dictamen que ponderaría los beneficios del tabaquismo contra sus riesgos cuando fuera posible aplicarlos a la población en general.

El resumen del dictamen del Comité

Con base en el estudio prolongado y en la evaluación de muchas líneas de evidencia convergentes, el Comité establece el siguiente dictamen:

\section{El tabaquismo es un riesgo para la salud de importancia suficiente en los EUA para justificar las acciones terapéuticas apropiadas.}

\section{B. Comentarios y conclusiones pormenorizadas \\ (Guía para la parte II del informe)}

Todas las conclusiones que adoptó de manera formal el Comité se presentan al final de esta sección, en negritas para facilitar su consulta. En aras de la concisión, en este resumen se omiten la documentación y la mayor parte del análisis. Junto con la tabla de contenido que aparece al principio del artículo esta parte se considera una guía del informe.

\section{Química y carcinogenicidad del tabaco y del humo del tabaco}

Los condensados del humo del tabaco son cancerígenos cuando se prueban mediante la aplicación en la piel de ratones y conejos y por inyección subcutánea en ratas (capítulo 9, pp. 143-145). No se ha producido carcinoma broncogénico por la aplicación de extractos, humo o condensados de tabaco en el pulmón o el árbol traqueobronquial de animales experimentales, salvo posiblemente en el caso de los perros (capítulo 9, p. 165).

Se generó carcinoma broncogénico en animales de laboratorio por la administración de hidrocarburos aromáticos policíclicos, ciertos metales, sustancias radiactivas y virus. Las características histopatológicas de los tumores producidos resultan similares a las que se observan en el hombre y son predominantemente de la variedad escamosa (capítulo 9, p. 166-167).

Se ha establecido que siete compuestos de hidrocarburos policíclicos aislados del humo del cigarro son cancerígenos en animales de laboratorio. Los resultados de varios ensayos de la cancerogenicidad de alquitranes del humo del tabaco presentan una anomalía incomprensible: el alquitrán total del cigarro tiene muchas veces la potencia cancerígena de la 
benzo(a)pirina presente en el primero. Los otros cancerígenos que se sabe están presentes en el humo del tabaco son, con excepción de la dibenzo(a,i)pirina, mucho menos potentes que la benzo(a)pirina y se encuentran en cantidades más pequeñas. Por lo tanto, en apariencia, el todo es mayor que la suma de las partes conocidas, discrepancia que tal vez se debe a la presencia de cocancerígenos en el humo del tabaco, o al daño en la producción de moco y en el mecanismo de transporte ciliar (capítulo 6, p. 61; capítulo 9, p. 144; y capítulo 10, pp. 267-269).

Hay abundantes evidencias de que la exposición industrial al hollín, al alquitrán del carbón, a las resinas y a los aceites minerales puede inducir el cáncer de piel en el hombre. La totalidad de estos compuestos contiene varios hidrocarburos aromáticos policíclicos que han demostrado ser cancerígenos en muchas especies de animales. Algunos de estos hidrocarburos se presentan también en el humo del tabaco, de modo que es razonable suponer que éstos igualmente pueden resultar cancerígenos para el hombre (capítulo 9, pp. 146-148).

Los factores genéticos desempeñan un papel importante en la formación de adenomas pulmonares en ratones, y es posible que influyan en el hábito del tabaquismo y en la respuesta en el hombre a los cancerígenos en el humo. Sin embargo, no hay evidencia de que hayan desempeñado un papel apreciable en el gran aumento de cáncer de pulmón en los humanos desde el inicio del siglo XX (capítulo 9, p. 190).

Se ha comprobado que los componentes de la fase gaseosa del humo del cigarro producen varios efectos indeseables en animales $u$ órganos de prueba, entre ellos la inhibición de la actividad de transporte ciliar, que es una función importante de limpieza en la tráquea y en los bronquios (capítulo 6, p. 61 y capítulo 10, pp. 267-270).

\section{Caracterización del hábito del tabaco}

El consumo habitual del tabaco se relaciona sobre todo con impulsos psicológicos y sociales, reforzados y perpetuados por los efectos farmacológicos de la nicotina sobre el sistema nervioso central. El tabaco sin nicotina, $\mathrm{u}$ otros constituyentes de la planta, no satisfacen las necesidades de aquellos que adquieren el hábito del tabaquismo (capítulo 13, p. 354).

\section{Patología y morfología}

Varios tipos de cambios epiteliales son mucho más comunes en la tráquea y los bronquios de los fumadores de cigarro, con o sin tumor maligno de pulmón, que en el caso de los no fumadores y pacientes sin ese padecimiento. Los cambios epiteliales incluyen: a) pérdida de cilios, b) hiperplasia de células basales y c) aparición de células atípicas con núcleos hipercromáticos irregulares; el grado de cada uno de los cambios epiteliales aumenta en general con el número de cigarros que se fuman, y se observan con mayor frecuencia cambios atípicos extensos en hombres que consumen dos o más cajetillas diarias.

Las mujeres fumadoras de cigarro, en general, tienen los mismos cambios epiteliales que los hombres fumadores. Sin embargo, a determinados niveles de consumo de cigarro, se manifiestan menos células atípicas en las mujeres que en los hombres. Los fumadores de mayor edad tienen más de estas células que los fumadores jóvenes. Los hombres que fuman pipa o cigarro tienen más cambios epiteliales que los no fumadores, pero menos que los fumadores de cigarro que consumen casi la misma cantidad de tabaco. Los varones ex fumadores de cigarro sufren de menos hiperplasia y de menor número de células atípicas que los que siguen fumando.

Es posible concluir, con base en las evidencias humana y experimental, que probablemente son malignas algunas de las lesiones hiperplásticas epiteliales en estado avanzado con muchas células atípicas, como se observa en los fumadores de cigarro (capítulo 9, pp. 167-173).

Clasificación de tumores. Los carcinomas escamosos y de célula oval (grupo I de la clasificación de Kreyberg) abarcan los tipos predominantes que se asocian con el aumento del tumor maligno de pulmón en la población masculina. En varios estudios, los adenocarcinomas (grupo II) han mostrado también un aumento definido, si bien en un grado mucho menor. La clasificación histológica del tumor maligno de pulmón es confiable, aunque el uso de la proporción de tipos histológicos como índice de la magnitud del aumento de éste es de valor limitado (capítulo 9, p. 173-175).

Cambios funcionales y patológicos. El tabaquismo produce alteraciones funcionales significativas en la tráquea, los bronquios y el pulmón. Al igual que otros agentes más, el consumo de cigarros puede reducir o anular la movilidad ciliar en animales de experimentación. El examen postmortem de bronquios de fumadores muestra una disminución en el número de células ciliadas, lo que reduce la cilia restante y cambia las células de copa y las glándulas mucosas. La posibilidad de deterioro funcional constituye la implicación de estas observaciones morfológicas.

En experimentos con animales, el tabaquismo afecta las características físicas de la capa que reviste los pulmones y deteriora la estabilidad alveolar (alvéolos). 
Los fagocitos alveolares ingieren componentes del humo del tabaco y ayudan en las tareas para removerlos del pulmón, mecanismo de limpieza que se interrumpe por la prolongada exposición de alto nivel al humo del cigarro: los componentes del humo se acumulan en los pulmones de los animales de los experimentos (capítulo 10, pp. 269-270).

Los efectos crónicos del tabaquismo sobre la función pulmonar se manifiestan por una reducción en la función ventilatoria, que se mide por el volumen espiratorio involuntario (capítulo 10, pp. 289-292).

Las alteraciones histopatológicas ocurren como consecuencia de la exposición al humo del tabaco por el árbol traqueobronquial y el parénquima pulmonar del hombre. Los cambios que ocurren regularmente en la bronquitis crónica -aumento del número de células de copa, hipertrofia e hiperplasia de las glándulas mucosas bronquiales- se presentan más a menudo en los bronquios de fumadores que en los de no fumadores. El tabaquismo produce alteraciones funcionales importantes en las vías aéreas superiores e inferiores, y esto pudiera interferir con los mecanismos de limpieza de los pulmones.

Los cambios patológicos en el parénquima pulmonar, como la ruptura del septo alveolar y la fibrosis, tienen una asociación muy estrecha con la historia pasada del tabaquismo, aunque no es posible relacionarlos de manera cierta con el enfisema y otras enfermedades reconocidas en la actualidad (capítulo 10, pp. 270-275).

\section{Mortalidad}

La tasa de muerte para fumadores de cigarros exclusivamente, que lo hacían en el momento de incorporarse en el estudio prospectivo particular, es casi $70 \%$ más alta que la de los no fumadores. Las tasas de muerte aumentan con la cantidad que se fuma. En grupos de hombres que fuman menos de 10, 10-19, 20-39 y 40 o más cigarros diarios, las tasas de muerte son, respectivamente, 40, 70, 90 y 120\% mayores que las de los no fumadores. La proporción de tasas de muerte entre fumadores y no fumadores es más alta en las edades más tempranas (40-50) representadas en estos estudios, y disminuye con el aumento de la edad. El mismo efecto parece mantenerse para la proporción entre la tasa de muerte de fumadores empedernidos y la de fumadores moderados. En los estudios que proporcionan esta información, la tasa de mortalidad de fumadores de cigarro, respecto a la de no fumadores, fue bastante superior para quienes empezaron a fumar antes de los 20 años que la de los que lo hicieron después de los
25. La tasa de mortalidad se incrementó a medida que aumentó el número de años en los que se practicó el tabaquismo. En dos estudios que registraron el grado de inhalación, la tasa de mortalidad para cierta cantidad de consumo de cigarros resultó mayor para inhaladores que para no inhaladores. Los fumadores que habían dejado el hábito antes de participar en el estudio tenían tasas de mortalidad cercanas a 1.4, en comparación con el 1.7 de los que seguían fumando. La tasa de mortalidad de los ex fumadores de cigarro aumentó con el número de años de tabaquismo, y resultó más alta para aquellos que dejaron el cigarro después de los 55 años que para los que se libraron de él a una edad menor (capítulo 8, p. 96).

Las tasas de muerte de fumadores de cigarro son casi las mismas que las correspondientes a no fumadores en hombres que consumen menos de cinco cigarros diarios. En el caso de hombres que fumaban cinco o más cigarros al día, las tasas de muerte resultaban un poco mayores (9 a $27 \%$ ) que para no fumadores en los cuatro estudios que proporcionaron esta información. Existen algunos indicios de que esta tasa de muerte más alta ocurre sobre todo en hombres que han fumado por más de 30 años y en hombres que han afirmado haber inhalado el humo hasta cierto grado. Las tasas de muerte de fumadores de pipa actuales fueron apenas, aunque no siempre, un poco superiores que para los no fumadores, incluso en el caso de hombres que fumaban 10 o más pipas llenas al día y en el de hombres que por más de 30 años fumaron con este aditamento. Por otra parte, ex fumadores de pipa y de cigarro presentaron tasas de muerte mayores, en comparación tanto con los no fumadores como con los que seguían fumando cigarro y pipa, en cuatro de cinco estudios (capítulo 8, p. 94). La explicación no es clara, pero es posible que un número considerable de estos fumadores dejó el hábito antes de enfermarse.

Mortalidad por causa de muerte. En los resultados combinados de los siete estudios prospectivos, la tasa de mortalidad de los fumadores de cigarro fue en particular alta en varias enfermedades. Hay un grupo adicional de ellas, que incluye algunas de las enfermedades crónicas más importantes, para las cuales la tasa de mortalidad correspondiente a los fumadores de cigarro se encuentra entre 1.2 y 2.0. No es clara la explicación de las elevaciones moderadas en las tasas de mortalidad en este grupo grande de causas. Una parte quizá se debe a las fuentes de propensión mencionadas antes o a alguna diferencia constitucional o genética entre fumadores y no fumadores de cigarro, además de existir la posibilidad de que el consumo de cigarro 
tenga algún efecto de debilitamiento general, aunque no es posible citar evidencia médica que apoye de manera clara esta hipótesis (capítulo 8, p. 105).

En los siete estudios, la enfermedad de las arterias coronarias es el contribuyente principal al número excesivo de fallecimientos de fumadores de cigarro en comparación con los no fumadores, con el cáncer de pulmón en forma constante en segundo lugar (capítulo 8, p. 108).

En el caso de fumadores que combinan cigarro y pipa, hubo una indicación de tasas de mortalidad elevadas respecto a tumores malignos de boca, esófago, laringe y pulmón, y úlceras duodenales y estomacales, si bien las tasas se sustentan en pocas muertes (capítulo 8, p. 107).

\section{Localización del cáncer}

Tumor maligno de pulmón

Fumar cigarro se relaciona de manera causal con el tumor maligno de pulmón en hombres; la magnitud del efecto del hábito del cigarro sobrepasa en importancia a los demás factores. Los datos en el caso de mujeres, aunque menos extensos, señalan hacia la misma dirección.

El riesgo de desarrollar cáncer de pulmón aumenta con la duración del hábito de fumar y con el número de cigarros diarios, y disminuye al interrumpir el consumo.

El riesgo de adquirir cáncer pulmonar para el grupo combinado de fumadores de pipa, fumadores de cigarro y fumadores de pipa y cigarro es mayor que para los no fumadores, pero mucho menor que para los fumadores sólo de cigarro. Los datos resultan insuficientes para asegurar una conclusión de manera individual para cada grupo (capítulo 9, p. 196).

\section{Tumor maligno de la cavidad oral}

Parece establecerse la relación causal de fumar en pipa $\mathrm{y}$ adquirir el tumor maligno de labio.

A pesar de que hay indicaciones de la relación entre el tumor maligno en otros sitios de la cavidad bucal y las diversas formas del uso del tabaco, sus implicaciones causales no pueden afirmarse en el presente (capítulo 9, pp. 204-205).

\section{Tumor maligno de la laringe}

La evaluación de la evidencia lleva al dictamen de que fumar cigarrillos es un factor importante en las causas del tumor maligno de laringe en los hombres (capítulo 9, p. 212).

Tumor maligno de esófago

La evidencia respecto a la relación tabaco-tumor maligno esofágico apoya la idea de que existe una asociación. Sin embargo, los datos no resultan suficientes para determinar si la relación es causal (capítulo 9, p. 218).

Tumor maligno de la vejiga urinaria

Los datos disponibles sugieren una asociación entre fumar cigarros y el tumor maligno de la vejiga urinaria en hombres, pero no son suficientes para apoyar un dictamente relativo a la importancia causal de dicha asociación (capítulo 9, p. 225).

Tumor maligno de estómago

No se ha establecido ninguna relación entre el uso de tabaco y el tumor maligno de estómago (capítulo 9, p. 229).

\section{Enfermedades respiratorias no neoplásicas, particularmente bronquitis crónica y enfisema pulmonar}

Fumar cigarros es la más importante de las causas de bronquitis crónica en los Estados Unidos de América, e incrementa el riesgo de morir a raíz de este padecimiento.

Existe una relación entre el enfisema pulmonar y el consumo de cigarrilos, pero no se ha establecido que sea causal. El hábito de fumar se vincula con un riesgo creciente de muerte por enfisema pulmonar.

Para el grueso de la población de los EUA, la importancia del consumo de cigarrillos como causa de enfermedad broncopulmonar crónica es mucho mayor que la de la contaminación atmosférica o la de las exposiciones ocupacionales.

La tos, la salivación, o las dos combinadas, son consistentemente más frecuentes entre fumadores de cigarrillos que entre no fumadores.

El hábito del cigarro se asocia con la reducción de la función ventilatoria. Entre hombres, los fumadores de cigarro sufren una mayor ocurrencia de insuficiencia respiratoria que los no fumadores.

El consumo de cigarros no parece provocar asma.

Si bien la certificación de muertes señala que los fumadores de cigarro tienen un riesgo moderadamente creciente de muerte por gripe y neumonía, no se justi- 
fica en otros casos una asociación entre el consumo de cigarros y las enfermedades infecciosas (capítulo 10, p. 302).

\section{Enfermedad cardiovascular}

El fumar y la administración de nicotina provocan efectos cardiovasculares agudos similares a los inducidos por la estimulación del sistema nervioso autónomo, aunque estos efectos no corresponden del todo a la asociación que se observa entre el hábito del cigarro y la enfermedad coronaria. Se establece que los hombres fumadores de cigarrillos tienen una tasa de muerte superior por enfermedad coronaria que los no fumadores. La asociación entre el tabaquismo y otros desórdenes cardiovasculares no está tan bien establecida. Si el cigarro causa en realidad la tasa de muertes más alta por enfermedad coronaria, sería responsable en este sentido del gran índice de fallecimientos de hombres de edad madura y de la tercera edad en los EUA. Se sabe también que otros factores como la hipertensión arterial, el alto colesterol y obesidad excesiva se asocian con la inusualmente alta tasa de muertes por enfermedades coronarias. Si bien no se ha probado el papel causal de estos factores en las enfermedades coronarias, se sospecha que son lo suficientemente importantes como para constituir una razón fundamental para emprender las medidas que los contrarresten. Resulta también más prudente asumir que la asociación establecida entre el consumo del cigarro y las enfermedades coronarias tiene un signficado causal, que suspender el dictamen hasta que no quede ninguna incertidumbre (capítulo 11, p. 327).

Los hombres fumadores de cigarrillos presentan una tasa de muerte superior por enfermedades de las arterias coronarias que la de los no fumadores, aunque no es claro que la asociación tenga un significado causal.

\section{Otras condiciones}

\section{Ulcera péptica}

Los estudios epidemiológicos indican una asociación entre el hábito del cigarrillo y la úlcera péptica, la cual es mayor para la gástrica que para la duodenal (capítulo 12, p. 340).

\section{Ambliopía por el tabaco}

La ambliopía por el tabaco (disminución de la visión que no se debe a una lesión orgánica) se ha vinculado con el fumado en pipa y con el de cigarro, pero la aso- ciación no se ha justificado con estudios epidemiológicos o experimentales (capítulo 12, p. 342).

\section{Cirrosis del hígado}

La mortalidad creciente de fumadores por causa de la cirrosis hepática se ha mostrado en estudios prospectivos, pero los datos no son suficientes para apoyar una asociación directa o causal (capítulo 12, p. 342).

\section{Tabaquismo en el embarazo y peso del bebé al nacer}

Las mujeres que fuman durante la preñez tienden a tener bebés de peso inferior; se carece de información acerca del mecanismo que provoca esta situación y se desconoce si la misma tiene alguna influencia en la buena salud biológica del recién nacido (capítulo 12, p. 343).

\section{Tabaquismo y accidentes}

El tabaquismo se asocia con muertes accidentales que resultan de incendios en el hogar.

No se cuenta con información concluyente sobre los efectos del tabaquismo en los accidentes de tráfico (capítulo 12, p. 345).

\section{Constitución morfológica de fumadores}

La evidencia disponible sugiere la existencia de algunas diferencias morfológicas entre fumadores y no fumadores, pero es demasiado pobre para permitir una conclusión (capítulo 15, p. 387).

\section{Aspectos sociales del tabaquismo}

No ha surgido una definición clara de la personalidad del fumador a partir de los resultados publicados hasta ahora. A pesar de que los fumadores difieren de los no fumadores en una diversidad de características, ninguno de los estudios ha exhibido una sola variable que se encuentre aislada en un grupo y esté completamente ausente en otro. Ni tampoco se ha confirmado una sola variable en una proporción suficientemente grande de fumadores, y en bastante pocos no fumadores, para considerarla un aspecto "esencial" del tabaquismo.

Las abundantes evidencias apuntan a la conclusión de que el tabaquismo -su inicio, hábito e interrupción ocasional- está en gran medida determinado psicológica y socialmente, lo cual no excluye factores fisiológicos, en especial respecto al hábito, ni la existencia de predisposición constitucional o factores hereditarios (capítulo 14, p. 377). 